The Canadian Mineralogist

Vol. 41, pp. 91-101 (2003)

\title{
THE CRYSTAL STRUCTURE OF BERGENITE, A NEW GEOMETRICAL ISOMER OF THE PHOSPHURANYLITE GROUP
}

\author{
ANDRew J. LOCOCK ${ }^{\S}$ AND Peter C. BURNS \\ Department of Civil Engineering and Geological Sciences, University of Notre Dame, \\ 156 Fitzpatrick Hall, Notre Dame, Indiana, 46556-5602, U.S.A.
}

\begin{abstract}
The crystal structure of bergenite, ideally $\mathrm{Ca}_{2} \mathrm{Ba}_{4}\left[\left(\mathrm{UO}_{2}\right)_{3} \mathrm{O}_{2}\left(\mathrm{PO}_{4}\right)_{2}\right]_{3}\left(\mathrm{H}_{2} \mathrm{O}\right)_{16}$, monoclinic, space group $P 2{ }_{1} / c, a 10.092(1), b$ 17.245(2), $c 17.355(2) \AA, \beta 113.678(2)^{\circ}, V 2766.2(3) \AA^{3}, Z=2, D_{\text {calc }} 4.82 \mathrm{~g} / \mathrm{cm}^{3}$, was solved by direct methods and refined by full-matrix least-squares techniques on the basis of $F^{2}$ to agreement indices $R 1$ of $5.0 \%$ calculated for 4118 unique observed reflections $\left(\left|F_{\mathrm{o}}\right| \geq 4 \sigma_{F}\right)$, and $w R_{2}$ of $11.9 \%$ for all data. Intensity data were collected at room temperature using MoK $\alpha$ radiation and a CCD-based area detector. The structure of bergenite contains five symmetrically independent uranium atoms, each of which is part of a $\left(\mathrm{UO}_{2}\right)^{2+}$ uranyl ion. The uranyl ions of $\mathrm{U}(1), \mathrm{U}(2)$ and $\mathrm{U}(3)$ are coordinated by five additional ligands giving pentagonal bipyramids, and the uranyl ions of $U(4)$ and $U(5)$ are coordinated by six additional ligands yielding hexagonal bipyramids. The uranyl pentagonal bipyramids share edges, forming dimers, which are linked to uranyl hexagonal bipyramids on either side, resulting in chains of bipyramids. Each uranyl hexagonal bipyramid shares two equatorial edges with phosphate tetrahedra, and the resulting uranyl phosphate chains are linked into sheets by the sharing of vertices of phosphate tetrahedra with uranyl pentagonal bipyramids of adjacent chains. The uranyl phosphate sheet in bergenite is a new geometrical isomer of the phosphuranylite group; the phosphate tetrahedra between the uranyl chains vary orientations in an up-up-down up-up-down (uuduud) pattern, and the pairs of tetrahedra attached to uranyl hexagonal bipyramids change orientations in a complementary same-same-opposite (SSO) manner (symbol uuduudSSO). In bergenite, the uranyl phosphate sheets are oriented parallel to (102), and the interlayer contains one calcium atom, two barium atoms and eight symmetrically distinct $\mathrm{H}_{2} \mathrm{O}$ groups.
\end{abstract}

Keywords: bergenite, phosphuranylite, uranyl phosphate, geometrical isomer, crystal structure.

\section{SOMMAIRE}

Nous avons résolu la structure cristalline de la bergenite, de formule idéale $\mathrm{Ca}_{2} \mathrm{Ba}_{4}\left[\left(\mathrm{UO}_{2}\right)_{3} \mathrm{O}_{2}\left(\mathrm{PO}_{4}\right)_{2}\right]_{3}\left(\mathrm{H}_{2} \mathrm{O}\right)_{16}$, monoclinique, groupe spatial $P 2_{1} / c, a 10.092(1), b 17.245(2), c 17.355(2) \AA, \beta 113.678(2)^{\circ}, V 2766.2(3) \AA^{3}, Z=2, D_{\text {calc }} 4.82 \mathrm{~g} / \mathrm{cm}^{3}, \mathrm{par}$ méthodes directes, et nous l'avons affinée par moindres carrés sur matrice entière en utilisant les facteurs $F^{2}$ jusqu'à un résidu $R 1$ de $5.0 \%$ calculé avec 4118 réflexions uniques observées $\left(\left|F_{\mathrm{o}}\right| \geq 4 \sigma_{F}\right)$, et $w R_{2}$ de $11.9 \%$ pour toutes les données. L'intensité des réflexions a été mesurée à température ambiante avec un rayonnement $\mathrm{MoK} \alpha$ et un détecteur à aire de type CCD. La structure de la bergenite contient cinq atomes d'uranium symétriquement indépendants, chacun faisant partie d'un ion uranyle, $\left(\mathrm{UO}_{2}\right)^{2+}$. Les ions uranyles $\mathrm{U}(1), \mathrm{U}(2)$ et $\mathrm{U}(3)$ ont des liaisons à cinq ligands additionnels, ce qui donne des bipyramides pentagonales, et les ions uranyles U(4) et U(5) ont des liaisons à six ligands additionnels, ce qui donne des bipyramides hexagonales. Les bipyramides pentagonales partagent des arêtes et forment ainsi des dimères, qui sont liés aux bipyramides uranylées hexagonales de chaque côté, pour donner des chaînes de bipyramides. Chaque bipyramide uranylée hexagonale partage deux arêtes équatoriales avec les tétraèdres de phosphate, et les chaînes de phosphate à uranyle qui en résultent sont agencées en feuillets par partage de vertex des tétraèdres de phosphate avec des dipyramides uranylées pentagonales des chaînes adjacentes. Le feuillet de phosphate uranylé dans la bergenite est un isomère géométrique nouveau du groupe de la phosphuranylite; les tétraèdres de phosphate entre les chaînes d'uranyle changent d'orientation selon la séquence haut-haut-bas haut-haut-bas (uuduud), et les paires de tétraèdres rattachés aux bipyramides uranylées hexagonales changent d'orientation de façon complémentaire: même-même-opposée (SSO) (symbole uuduudSSO). Dans la bergenite, les feuillets de phosphate à uranyle sont orientés parallèles à (102), et l'interfeuillet contient un atome de calcium, deux atomes de barium, et huit groupes $\mathrm{H}_{2} \mathrm{O}$ symétriquement distincts.

(Traduit par la Rédaction)

Mots-clés: bergenite, phosphuranylite, phosphate uranylé, isomère géométrique, structure cristalline.

§E-mail address: alocock@nd.edu 


\section{INTRODUCTION}

The phosphuranylite group is one of the two major groups of uranyl phosphate and uranyl arsenate minerals (autunite - meta-autunite is the other); it consists of at least sixteen minerals, of which nine have had their structures determined (Finch \& Murakami 1999, Burns 1999). The structures, chemical compositions and stabilities of uranyl phosphate minerals are of interest because of their significance to the environment. They exert an impact on the mobility of uranium in phosphaterich systems (Sowder et al. 1996), such as in the Koongarra uranium deposit in Australia (Murakami et al. 1997), the soils of the Fernald site in Ohio (Buck et al. 1996), and the K1300 locality of the DOE-K 25 site at Oak Ridge, Tennessee (Roh et al. 2000). As part of our ongoing research into the structures of uranyl phosphates (Locock \& Burns 2002a, b, 2003), we have determined the crystal structure of bergenite, the calcium-barium member of the phosphuranylite group.

\section{Previous Studies}

Bergenite was originally described by Bültemann \& Moh (1959) as a member of the phosphuranylite group; it was discovered in material from a now-vanished mine dump of the SDAG Wismut 254 shaft in the area of Streuberg, near Bergen on the Trieb, Vogtland, Sachsen, Federal Republic of Germany. Bergenite was reported to have orthorhombic symmetry, a density greater than $4.1 \mathrm{~g} / \mathrm{cm}^{3}$, and was interpreted to be the natural analogue of the synthetic "barium-phosphuranylite" of Ross (1956), $\mathrm{Ba}\left(\mathrm{UO}_{2}\right)_{4}\left(\mathrm{PO}_{4}\right)_{2}(\mathrm{OH})_{4} \bullet 8 \mathrm{H}_{2} \mathrm{O}$, on the basis of the similarities between the optical properties and the X-ray diffraction patterns of the two compounds (Bültemann \& Moh 1959). No quantitative chemical data were presented.

Piret \& Deliens (1981) re-investigated bergenite, using material from the holotype specimen, and concluded that although bergenite is a member of the phosphuranylite group, the equivalence of bergenite and synthetic "barium-phosphuranylite" is far from complete. They reported new optical data for bergenite, a chemical formula of $\mathrm{Ba}_{4 / 3} \mathrm{Ca}_{2 / 3}\left(\mathrm{UO}_{2}\right)_{3}\left(\mathrm{PO}_{4}\right)_{2}(\mathrm{OH})_{4}$ - 5.5 $\left(\mathrm{H}_{2} \mathrm{O}\right)$ based on electron-microprobe analyses, and the following crystallographic data: monoclinic, space group $P 2{ }_{1} / c, a 23.32, b 17.19, c 20.63 \AA, \beta 93.0^{\circ}, V$ $8259 \AA^{3}, Z=18, D_{\text {calc }} 4.98 \mathrm{~g} / \mathrm{cm}^{3}, D_{\text {meas }}>4.05 \mathrm{~g} / \mathrm{cm}^{3}$.

In addition to the Vogtland locality, bergenite has been found in the uranium deposits of the Black Forest, Federal Republic of Germany (Walenta 1985), and in the graphitic uranium ore of the Black Slate Formation of the Ogcheon Group in the Republic of [South] Korea (Lee et al. 1982).

\section{EXPERIMENTAL}

The bergenite sample studied is from the collection of L'Institut Royal des Sciences Naturelles de Belgique in Brussels, sample RV9/535. It was donated to the Institute by George Vanacker, who had originally obtained the specimen from William Pinch. The sample investigated is not part of the holotype, but is from the type locality.

\section{Electron-microprobe examination}

In light of the lack of agreement concerning the presence of K in phosphuranylite (Demartin et al. 1991, Piret $\&$ Piret-Meunier 1991), the chemical composition of bergenite was confirmed by qualitative electron-microprobe examination. Crystals of bergenite were mounted on an adhesive carbon tab and carbon-coated. An energy-dispersion X-ray emission spectrum was acquired for $140 \mathrm{~s}$ with an ultra-thin-window $\mathrm{Si}(\mathrm{Li})$ detector on a JEOL JXA-8600 Superprobe at an excitation voltage of $15 \mathrm{kV}$ and a probe current of $0.9 \mathrm{nA}$. Following EDS examination, a wavelength-dispersion X-ray emission spectrum was acquired in the energy range 3.05-3.45 $\mathrm{kV}$ (PET crystal, excitation voltage $15 \mathrm{kV}$, probe current $20 \mathrm{nA}$ ) to check for the presence of $\mathrm{K}$ and resolve the potential overlap of the $\mathrm{K} K \alpha$ peak with that of $\mathrm{U} M \beta$. The only elements present (Fig. 1a) were U, Ba, Ca, P, $\mathrm{O}$ and $\mathrm{C}$; $\mathrm{K}$ was not detected (Fig. 1b).

\section{Single-crystal X-ray diffraction}

An optically homogeneous fragment of bergenite was mounted on a Bruker PLATFORM three-circle Xray diffractometer operated at $50 \mathrm{keV}$ and $40 \mathrm{~mA}$ and equipped with a $4 \mathrm{~K}$ APEX CCD detector with a crystal-to-detector distance of $4.7 \mathrm{~cm}$. A sphere of threedimensional data was collected using graphitemonochromatized $\mathrm{MoK} \alpha \mathrm{X}$-radiation and frame widths of $0.6^{\circ}$ in $\omega$, with count-times per frame of 240 seconds. Data were collected in 84 hours for $4^{\circ} \leq 2 \theta \leq 69^{\circ}$; comparison of the intensities of equivalent reflections measured at different times during data acquisition showed no significant decay. The unit cell (Table 1) was refined with 3472 reflections using least-squares techniques; its volume is three times smaller than that reported by Piret \& Deliens (1981). The intensity data were examined carefully for indications of a larger cell, but no reflections with $I \geq 2 \sigma_{I}$ were found to support this possibility, despite the high sensitivity of the CCD detector (Burns 1998 ) and the long count-time per frame (240 s). The intensity data were reduced and corrected for Lorentz, polarization, and background effects using the Bruker program SAINT. A semi-empirical correction for 
I ABLE 1. CKYSI ALLUGKAYHIC DAIA AND DE I AILS UF IHE SI KUCIUKE REFINEMENT FOR BERGENITE

\begin{tabular}{|c|c|c|c|}
\hline $\begin{array}{l}a(\AA) \\
b(\AA)\end{array}$ & $\begin{array}{l}10.092(1) \\
17245(2)\end{array}$ & Crystal size & $\begin{array}{l}0.08 \mathrm{~mm} \times 0.06 \mathrm{~mm} \times 0.02 \mathrm{~mm} \\
16.3\end{array}$ \\
\hline$b(\AA)$ & $\begin{array}{l}17.245(2) \\
17.355(2)\end{array}$ & $R_{\text {int }}(\%)$ & $\begin{array}{l}10.5 \\
54037\end{array}$ \\
\hline$c(A)$ & $17.355(2)$ & Total reflections & 54,037 \\
\hline$\beta\left({ }^{\circ}\right)$ & $113.678(2)$ & Unique reflections & 11,359 \\
\hline$V\left(\AA^{3}\right)$ & $2766.2(3)$ & Unique $F_{\mathrm{o}} \geq 4 \sigma_{F}$ & 4118 \\
\hline Temperature (K) & $293(2)$ & Refinement method & Full-matrix least-squares on $F^{2}$ \\
\hline Space group & $P 2_{1} / c$ & Parameters varied & 227 \\
\hline$F(000)^{*}$ & 3452.0 & $R 1 \dagger(\%)$ for $F_{\mathrm{o}} \geq 4 \sigma_{F}$ & 5.0 \\
\hline$\mu\left(\mathrm{mm}^{-1}\right)^{*}$ & 29.53 & $w R_{2} \ddagger(\%)$ all data & 11.9 \\
\hline$D_{\text {calc }}\left(\mathrm{g} / \mathrm{cm}^{3}\right)^{*}$ & $4.82(1)$ & Goodness of fit all data & 0.777 \\
\hline Wavelength & $\operatorname{MoK} \alpha$ & Max. min. peaks $\left(e / \AA^{3}\right)$ & $4.9,-2.9$ \\
\hline Data range $(\theta)$ & 2.20 to $34.56^{\circ}$ & Data collected & $-16 \leq h \leq 15,-27 \leq k \leq 27,-27 \leq l \leq 27$ \\
\hline Formula weight* & 4013.78 & Formula $(Z=2)$ & $\mathrm{Ca}_{2} \mathrm{Ba}_{4}\left[\left(\mathrm{UO}_{2}\right)_{3} \mathrm{O}_{2}\left(\mathrm{PO}_{4}\right)_{2}\right]_{3}\left(\mathrm{H}_{2} \mathrm{O}\right)_{16}$ \\
\hline
\end{tabular}

* Calculated with ideal occupancy of all atomic positions

$\dagger R 1=\left[\Sigma F_{\mathrm{o}}-F_{\mathrm{c}}\right] / \boldsymbol{\Sigma} F_{\mathrm{o}} \times 100$

$\ddagger w R_{2}=\left[\Sigma\left[w\left(F_{\mathrm{o}}^{2}-F_{\mathrm{c}}^{2}\right)^{2}\right] / \Sigma\left[w\left(F_{\mathrm{o}}^{2}\right)^{2}\right]\right]^{0.5} \times 100, w=1 /\left(\sigma^{2}\left(F_{\mathrm{o}}{ }^{2}\right)+(a \cdot P)^{2}\right.$,

$P=1 / 3 \max \left(0, F_{\mathrm{o}}^{2}\right)+2 / 3 F_{\mathrm{c}}^{2}, a=0.0372$
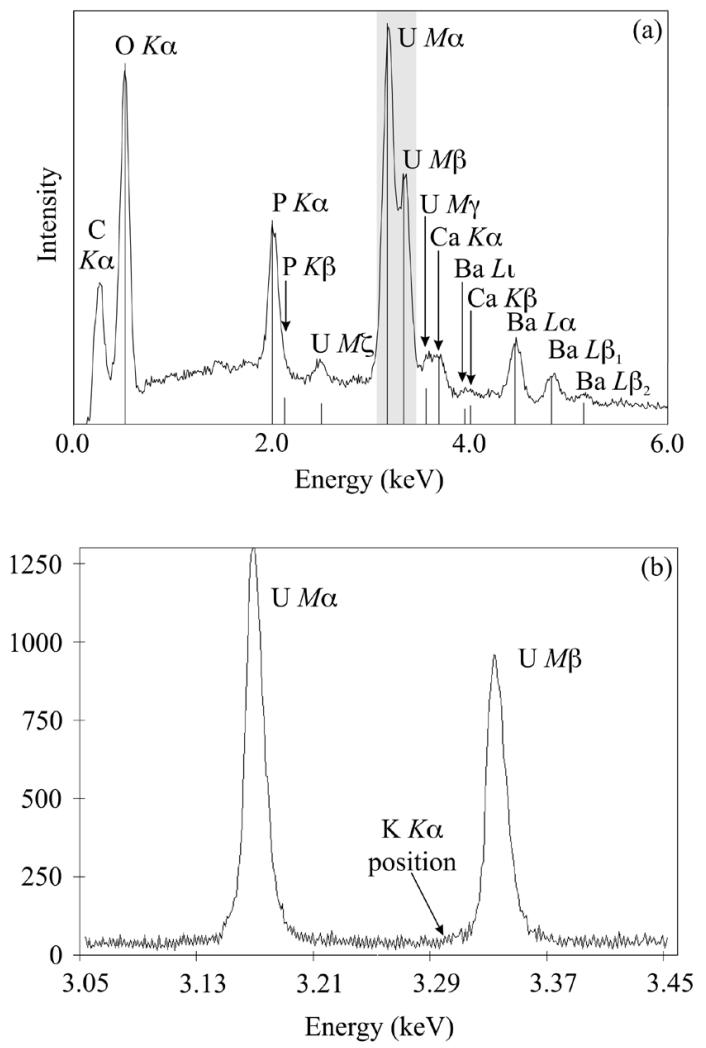

FIG. 1. (a) Energy-dispersion X-ray emission spectrum of bergenite; the shaded area corresponds to the energy range investigated by WDS. (b) Wavelength-dispersion X-ray emission spectrum of bergenite; the $\mathrm{U} M \alpha$ and $\mathrm{U} M \beta$ lines are well resolved, and $\mathrm{K} K \alpha$ is not detected. absorption was applied by modeling the crystal as an ellipsoid; this lowered $R_{\mathrm{INT}}$ of 1916 intense reflections from $8.3 \%$ to $6.2 \%$.

Systematic absences of reflections for bergenite were found to be consistent with space group $P 2_{1} / c$. A total of 54,917 intensities was collected, of which 880 were discarded as being inconsistent with the space group; none of these latter intensities were classified as observed. Of the 54,037 remaining intensities, 11,359 are unique $\left(R_{\mathrm{INT}}=16.3 \%\right)$, of which 4118 are classified as observed reflections $\left(\left|F_{\mathrm{o}}\right| \geq 4 \sigma_{F}\right)$.

Scattering curves for neutral atoms, together with anomalous dispersion corrections, were taken from International Tables for X-ray Crystallography, Volume IV (Ibers \& Hamilton 1974). The Bruker SHELXTL Version 5 series of programs was used for the solution and refinement of the crystal structure.

\section{Structure solution and refinement}

The structure of bergenite was solved by direct methods and was refined successfully on the basis of $F^{2}$ for all unique data in space group $P 2_{1} / c$. A structure model including anisotropic displacement parameters for all non-O atoms converged, and gave an agreement index (R1) of $5.0 \%$, calculated for the 4118 observed unique reflections $\left(\left|F_{\mathrm{o}}\right| \geq 4 \sigma_{F}\right)$. The final value of $w R_{2}$ was $11.9 \%$ for all data using the structure-factor weights assigned during least-squares refinement. In the final cycle of refinement, the mean parameter shift/esd was 0.000 , and the maximum peaks in the final differenceFourier maps were 4.8 and $-2.9 e / \AA^{3}$. The locations of the $\mathrm{H}$ atoms in the unit cell were not determined. The positional parameters and anisotropic displacement parameters of atoms in bergenite are given in Tables 2 and 3 respectively, and selected interatomic distances are in Table 4. Observed and calculated structure-factors are 
available from the Depository of Unpublished Data, CISTI, National Research Council, Ottawa, Ontario K1A 0S2, Canada. Bond-valence sums at the cation and anion sites are presented in Table 5, and were calculated assuming ideal occupancy of all cation sites and using the parameters of Burns et al. (1997) for U, and

TABLE 2 ATOM COORDINATES AND

ISOTROPIC DISPLACEMENT PARAMETERS $\left(\AA^{2}\right)$ FOR BERGENITE

\begin{tabular}{|c|c|c|c|c|}
\hline & $x$ & $y$ & $z$ & $U_{\mathrm{eq}}$ \\
\hline $\begin{array}{l}\mathrm{U}(1) \\
\mathrm{U}(2) \\
\mathrm{U}(3) \\
\mathrm{U}(4) \\
\mathrm{U}(5)\end{array}$ & $\begin{array}{l}0.3119(1) \\
0.3234(1) \\
1.0002(1) \\
0 \\
0.6382(1)\end{array}$ & $\begin{array}{l}0.3921(1) \\
0.6086(1) \\
0.3929(1) \\
1 / 2 \\
0.5023(1)\end{array}$ & $\begin{array}{l}0.6823(1) \\
0.6779(1) \\
0.9942(1) \\
1 / 2 \\
0.8603(1)\end{array}$ & $\begin{array}{l}0.012(1) \\
0.013(1) \\
0.012(1) \\
0.016(1) \\
0.014(1)\end{array}$ \\
\hline $\begin{array}{l}\mathrm{Ba}(1)^{*} \\
\mathrm{Ba}(2)^{\dagger} \\
\mathrm{Ca}(1)\end{array}$ & $\begin{array}{l}0.2494(1) \\
0.3888(1) \\
0.0538(4)\end{array}$ & $\begin{array}{l}0.4880(1) \\
0.6584(1) \\
0.6942(2)\end{array}$ & $\begin{array}{l}0.8786(1) \\
0.4582(1) \\
0.7974(2)\end{array}$ & $\begin{array}{l}0.026(1) \\
0.024(1) \\
0.025(1)\end{array}$ \\
\hline $\begin{array}{l}\mathrm{P}(1) \\
\mathrm{P}(2) \\
\mathrm{P}(3)\end{array}$ & $\begin{array}{l}0.6278(4) \\
0.6239(5) \\
0.0352(4)\end{array}$ & $\begin{array}{l}0.3099(2) \\
0.6927(2) \\
0.6885(2)\end{array}$ & $\begin{array}{l}0.8703(3) \\
0.8719(3) \\
0.4729(3)\end{array}$ & $\begin{array}{l}0.013(1) \\
0.014(1) \\
0.016(1)\end{array}$ \\
\hline $\begin{array}{l}\mathrm{O}(1) \\
\mathrm{O}(2) \\
\mathrm{O}(3) \\
\mathrm{O}(4) \\
\mathrm{O}(5) \\
\mathrm{O}(6) \\
\mathrm{O}(7) \\
\mathrm{O}(8) \\
\mathrm{O}(9) \\
\mathrm{O}(10) \\
\mathrm{O}(11) \\
\mathrm{O}(12) \\
\mathrm{O}(13) \\
\mathrm{O}(14) \\
\text { O(15) } \\
\text { O(16) } \\
\text { O(17) } \\
\text { O(18) } \\
\text { OW(19) } \\
\mathrm{O}(20) \\
\text { O(21) } \\
\text { O(22) } \\
\text { OW(23) } \\
\text { O(24) } \\
\text { O(25) } \\
\text { O(26) } \\
\text { OW(27) } \\
\text { OW(28) } \\
\text { OW(29) } \\
\text { OW(30) } \\
\text { OW(31) } \\
\text { OW(32) }\end{array}$ & $\begin{array}{c}0.2043(10) \\
0.4480(12) \\
0.4360(13) \\
0.0908(13) \\
0.1964(11) \\
0.7167(12) \\
0.5653(10) \\
0.6646(11) \\
0.1997(12) \\
0.6792(11) \\
0.5270(11) \\
0.8576(10) \\
-0.0998(11) \\
0.4101(9) \\
0.9747(12) \\
0.0050(11) \\
0.5064(12) \\
1.0265(11) \\
0.3159(12) \\
0.7534(12) \\
0.7463(10) \\
0.5912(13) \\
0.3016(12) \\
0.5425(13) \\
0.0821(13) \\
0.1529(12) \\
-0.2083(15) \\
-0.0199(13) \\
-0.0588(15) \\
0.2215(14) \\
0.6613(17) \\
-0.2998(16)\end{array}$ & $\begin{array}{l}0.5019(6) \\
0.5990(6) \\
0.3967(7) \\
0.4872(7) \\
0.3862(6) \\
0.5076(7) \\
0.5001(6) \\
0.2457(5) \\
0.6232(6) \\
0.7554(6) \\
0.6351(6) \\
0.4999(6) \\
0.6350(6) \\
0.5018(6) \\
0.3862(6) \\
0.7569(5) \\
0.3623(6) \\
0.3996(6) \\
0.3322(6) \\
0.3692(6) \\
0.6389(5) \\
0.2769(7) \\
0.6498(6) \\
0.7262(6) \\
0.7139(6) \\
0.6373(6) \\
0.6712(8) \\
0.5349(7) \\
0.7430(8) \\
0.8029(7) \\
0.4891(9) \\
0.6375(8)\end{array}$ & $\begin{array}{l}0.6131(6) \\
0.6280(7) \\
0.6314(8) \\
0.4289(7) \\
0.7381(7) \\
0.7846(7) \\
0.9394(6) \\
0.8206(6) \\
0.7284(7) \\
0.8307(6) \\
0.8051(7) \\
0.9664(6) \\
0.4430(6) \\
0.7635(6) \\
1.0906(7) \\
0.5179(6) \\
0.8113(7) \\
0.8984(6) \\
0.9160(7) \\
0.9070(7) \\
0.9280(6) \\
0.9389(8) \\
0.9093(7) \\
0.9207(7) \\
0.4042(7) \\
0.5384(7) \\
0.7411(9) \\
0.7490(8) \\
0.6558(8) \\
0.7858(8) \\
0.6064(10) \\
0.5629(9)\end{array}$ & $\begin{array}{l}0.019(2) \\
0.025(3) \\
0.033(3) \\
0.034(3) \\
0.021(2) \\
0.034(3) \\
0.022(2) \\
0.013(2) \\
0.025(3) \\
0.016(3) \\
0.020(3) \\
0.021(2) \\
0.019(2) \\
0.017(2) \\
0.029(3) \\
0.016(2) \\
0.027(3) \\
0.020(2) \\
0.024(3) \\
0.026(3) \\
0.015(2) \\
0.036(3) \\
0.024(3) \\
0.030(3) \\
0.029(3) \\
0.029(3) \\
0.054(4) \\
0.035(3) \\
0.047(4) \\
0.041(3) \\
0.071(5) \\
0.059(4)\end{array}$ \\
\hline
\end{tabular}

$U_{\mathrm{eq}}$ is defined as one third of the trace of the orthogonalized $U_{i j}$ tensor.

$*$ eqa(1) refined occupancy: $\mathrm{Ba} 0.968(6), \mathrm{Ca} 0.032(6)$

$\uparrow \mathrm{Ba}(2)$ refined occupancy: $\mathrm{Ba} 0.879(5), \mathrm{Ca} 0.121(5)$

TABLE 3. ANISOTROPIC DISPLACEMENT PARAMETERS $\left(\AA^{2}\right)$ FOR BERGENITE

\begin{tabular}{lcccrrr}
\hline & $U_{11}$ & $U_{22}$ & $U_{33}$ & \multicolumn{1}{c}{$U_{23}$} & \multicolumn{1}{c}{$U_{13}$} & \multicolumn{1}{c}{$U_{12}$} \\
\hline $\mathrm{U}(1)$ & $0.011(1)$ & $0.006(1)$ & $0.014(1)$ & $-0.001(1)$ & $-0.002(1)$ & $0.001(1)$ \\
$\mathrm{U}(2)$ & $0.011(1)$ & $0.007(1)$ & $0.015(1)$ & $0.000(1)$ & $-0.001(1)$ & $0.000(1)$ \\
$\mathrm{U}(3)$ & $0.008(1)$ & $0.007(1)$ & $0.017(1)$ & $0.000(1)$ & $0.001(1)$ & $-0.001(1)$ \\
$\mathrm{U}(4)$ & $0.015(1)$ & $0.011(1)$ & $0.015(1)$ & $0.001(1)$ & $-0.002(1)$ & $0.001(1)$ \\
$\mathrm{U}(5)$ & $0.010(1)$ & $0.012(1)$ & $0.016(1)$ & $0.001(1)$ & $0.001(1)$ & $0.001(1)$ \\
$\mathrm{Ba}(1)$ & $0.022(1)$ & $0.019(1)$ & $0.031(1)$ & $-0.004(1)$ & $0.006(1)$ & $-0.006(1)$ \\
$\mathrm{Ba}(2)$ & $0.030(1)$ & $0.015(1)$ & $0.025(1)$ & $-0.002(1)$ & $0.009(1)$ & $0.001(1)$ \\
$\mathrm{Ca}(1)$ & $0.030(2)$ & $0.019(2)$ & $0.029(2)$ & $-0.005(1)$ & $0.016(2)$ & $-0.008(1)$ \\
$\mathrm{P}(1)$ & $0.007(2)$ & $0.003(2)$ & $0.019(2)$ & $0.000(2)$ & $-0.004(2)$ & $0.001(2)$ \\
$\mathrm{P}(2)$ & $0.016(2)$ & $0.002(2)$ & $0.018(2)$ & $0.003(2)$ & $0.001(2)$ & $-0.001(2)$ \\
$\mathrm{P}(3)$ & $0.014(2)$ & $0.002(2)$ & $0.021(2)$ & $-0.001(1)$ & $-0.003(2)$ & $-0.002(1)$ \\
\end{tabular}

The anisotropic displacement parameter exponent takes the form: $-2 \pi^{2}\left[h^{2} \mathbf{a}^{*^{2}} U_{u}+\right.$ $\left.\ldots+2 h k \mathbf{a}^{\star} \mathbf{b}^{\star} U_{12}\right]$ those of Brown \& Altermatt (1985) for P, Ba and Ca. Delineation of the $\mathrm{H}_{2} \mathrm{O}$ groups was accomplished using the results of the bond-valence analysis.

The site occupancies (Hawthorne et al. 1995) of the $\mathrm{Ba}(1)$ and $\mathrm{Ba}(2)$ sites were refined, assuming no vacancies and only $\mathrm{Ca}$ ö Ba substitution, consistent with the qualitative elemental data presented herein (Fig. 1) and the quantitative electron-microprobe results of Piret \&

TABLE 4. SELECTED INTERATOMIC DISTANCES $(\AA)$ FOR BERGENITE

\begin{tabular}{|c|c|c|c|}
\hline $\mathrm{U}(1)-\mathrm{O}(5)$ & $1.792(11)$ & $\mathrm{P}(1)-\mathrm{O}(22)$ & $1.493(13)$ \\
\hline $\mathrm{U}(1)-\mathrm{O}(3)$ & $1.802(12)$ & $\mathrm{P}(1)-\mathrm{O}(17)$ & $1.536(11)$ \\
\hline $\mathrm{U}(1)-\mathrm{O}(1)$ & $2.271(10)$ & $\mathrm{P}(1)-\mathrm{O}(8)$ & $1.539(10)$ \\
\hline $\mathrm{U}(1)-\mathrm{O}(14)$ & $2.328(10)$ & $\mathrm{P}(1)-\mathrm{O}(20)$ & $1.554(11)$ \\
\hline $\mathrm{U}(1)-\mathrm{O}(17)$ & $2.366(11)$ & $<\mathrm{P}(1)-\mathrm{O}>$ & 1.53 \\
\hline $\mathrm{U}(1)-\mathrm{O}(10) \mathrm{a}$ & $2.374(10)$ & & \\
\hline $\mathrm{U}(1)-\mathrm{O}(13) \mathrm{b}$ & $2.407(10)$ & $\mathrm{P}(2)-\mathrm{O}(24)$ & $1.510(12)$ \\
\hline$<\mathrm{U}(1)-\mathrm{O}_{\mathrm{ur}}>$ & 1.80 & $\mathrm{P}(2)-\mathrm{O}(10)$ & $1.520(11)$ \\
\hline \multirow{2}{*}{$<\mathrm{U}(1)-\mathrm{O}_{\mathrm{eq}}>$} & 2.35 & $\mathrm{P}(2)-\mathrm{O}(21)$ & $1.539(10)$ \\
\hline & & $\mathrm{P}(2)-\mathrm{O}(11)$ & $1.541(11)$ \\
\hline $\mathrm{U}(2)-\mathrm{O}(2)$ & $1.797(11)$ & $<\mathrm{P}(2)-\mathrm{O}>$ & 1.53 \\
\hline $\mathrm{U}(2)-\mathrm{O}(9)$ & $1.809(11)$ & & \\
\hline $\mathrm{U}(2)-\mathrm{O}(1)$ & $2.238(10)$ & $\mathrm{P}(3)-\mathrm{O}(16)$ & $1.512(10)$ \\
\hline $\mathrm{U}(2)-\mathrm{O}(14)$ & $2.307(10)$ & $\mathrm{P}(3)-\mathrm{O}(25)$ & $1.514(12)$ \\
\hline $\mathrm{U}(2)-\mathrm{O}(8) \mathrm{c}$ & $2.366(10)$ & $\mathrm{P}(3)-\mathrm{O}(26)$ & $1.548(12)$ \\
\hline $\mathrm{U}(2)-\mathrm{O}(11)$ & $2.380(10)$ & $\mathrm{P}(3)-\mathrm{O}(13)$ & $1.552(11)$ \\
\hline $\mathrm{U}(2)-\mathrm{O}(26)$ & $2.392(11)$ & $<\mathrm{P}(3)-\mathrm{O}>$ & 1.53 \\
\hline$<\mathrm{U}(2)-\mathrm{O}_{\mathrm{ur}}>$ & 1.79 & & \\
\hline \multirow{2}{*}{$<\mathrm{U}(2)-\mathrm{O}_{\mathrm{eq}}>$} & 2.34 & $\mathrm{Ba}(1)-\mathrm{OW}(19)$ & $2.783(11)$ \\
\hline & & $\mathrm{Ba}(1)-\mathrm{OW}(23)$ & $2.850(11)$ \\
\hline $\mathrm{U}(3)-\mathrm{O}(18)$ & $1.789(10)$ & $\mathrm{Ba}(1)-\mathrm{O}(18) \mathrm{e}$ & $2.851(10)$ \\
\hline $\mathrm{U}(3)-\mathrm{O}(15)$ & $1.796(11)$ & $\mathrm{Ba}(1)-\mathrm{OW}(28)$ & $2.862(12)$ \\
\hline $\mathrm{U}(3)-\mathrm{O}(12)$ & $2.270(11)$ & $\mathrm{Ba}(1)-\mathrm{O}(5)$ & $2.876(11)$ \\
\hline $\mathrm{U}(3)-\mathrm{O}(12) \mathrm{d}$ & $2.270(11)$ & $\mathrm{Ba}(1)-\mathrm{O}(7)$ & $2.935(9)$ \\
\hline $\mathrm{U}(3)-\mathrm{O}(16) \mathrm{a}$ & $2.354(9)$ & $\mathrm{Ba}(1)-\mathrm{O}(7) \mathrm{f}$ & $2.962(9)$ \\
\hline $\mathrm{U}(3)-\mathrm{O}(20)$ & $2.373(11)$ & $\mathrm{Ba}(1)-\mathrm{O}(14)$ & $3.046(9)$ \\
\hline $\mathrm{U}(3)-\mathrm{O}(21) \mathrm{d}$ & $2.428(10)$ & $\mathrm{Ba}(1)-\mathrm{O}(12) \mathrm{f}$ & $3.284(9)$ \\
\hline$<\mathrm{U}(3)-\mathrm{O}_{\mathrm{ur}}>$ & 1.79 & $\mathrm{Ba}(1)-\mathrm{O}(15) \mathrm{f}$ & $3.329(11)$ \\
\hline \multirow{2}{*}{$<\mathrm{U}(3)-\mathrm{O}_{\mathrm{eq}}>$} & 2.27 & $\mathrm{Ba}(1)-\mathrm{O}(9)$ & $3.381(11)$ \\
\hline & & $<\mathrm{Ba}(1)-\varphi>$ & 3.01 \\
\hline $\mathrm{U}(4)-\mathrm{O}(4)$ & $1.821(11)$ & & \\
\hline $\mathrm{U}(4)-\mathrm{O}(4) \mathrm{b}$ & $1.821(11)$ & $\mathrm{Ba}(2)-\mathrm{O}(22) \mathrm{c}$ & $2.668(12)$ \\
\hline $\mathrm{U}(4)-\mathrm{O}(1) \mathrm{b}$ & $2.204(9)$ & $\mathrm{Ba}(2)-\mathrm{OW}(31) \mathrm{g}$ & $2.743(16)$ \\
\hline $\mathrm{U}(4)-\mathrm{O}(1)$ & $2.204(9)$ & $\mathrm{Ba}(2)-\mathrm{O}(24) \mathrm{h}$ & $2.756(12)$ \\
\hline $\mathrm{U}(4)-\mathrm{O}(13)$ & $2.572(10)$ & $\mathrm{Ba}(2)-\mathrm{OW}(30) \mathrm{h}$ & $2.862(13)$ \\
\hline $\mathrm{U}(4)-\mathrm{O}(13) \mathrm{b}$ & $2.572(10)$ & $\mathrm{Ba}(2)-\mathrm{O}(3) \mathrm{g}$ & $2.942(12)$ \\
\hline $\mathrm{U}(4)-\mathrm{O}(26)$ & $2.757(11)$ & $\mathrm{Ba}(2)-\mathrm{O}(2)$ & $2.947(11)$ \\
\hline $\mathrm{U}(4)-\mathrm{O}(26) \mathrm{b}$ & $2.757(11)$ & $\mathrm{Ba}(2)-\mathrm{OW}(32) \mathrm{i}$ & $2.954(15)$ \\
\hline$<\mathrm{U}(4)-\mathrm{O}_{\mathrm{ur}}>$ & 1.82 & $\mathrm{Ba}(2)-\mathrm{O}(25)$ & $3.007(12)$ \\
\hline \multirow[t]{2}{*}{$<\mathrm{U}(4)-\mathrm{O}_{\mathrm{eq}}>$} & 2.51 & $\mathrm{Ba}(2)-\mathrm{O}(26)$ & $3.225(11)$ \\
\hline & & $\mathrm{Ba}(2)-\mathrm{OW}(23) \mathrm{h}$ & $3.440(11)$ \\
\hline $\mathrm{U}(5)-\mathrm{O}(6)$ & $1.789(11)$ & $<\mathrm{Ba}(2)-\varphi>$ & 2.95 \\
\hline $\mathrm{U}(5)-\mathrm{O}(7)$ & $1.799(9)$ & & \\
\hline $\mathrm{U}(5)-\mathrm{O}(14)$ & $2.240(9)$ & $\mathrm{Ca}(1)-\mathrm{O}(25) \mathrm{j}$ & $2.367(12)$ \\
\hline $\mathrm{U}(5)-\mathrm{O}(12)$ & $2.240(9)$ & $\mathrm{Ca}(1)-\mathrm{OW}(29)$ & $2.408(14)$ \\
\hline $\mathrm{U}(5)-\mathrm{O}(20)$ & $2.554(11)$ & $\mathrm{Ca}(1)-\mathrm{OW}(27)$ & $2.456(15)$ \\
\hline $\mathrm{U}(5)-\mathrm{O}(11)$ & $2.561(10)$ & $\mathrm{Ca}(1)-\mathrm{O}(15) \mathrm{f}$ & $2.496(12)$ \\
\hline $\mathrm{U}(5)-\mathrm{O}(21)$ & $2.662(10)$ & $\mathrm{Ca}(1)-\mathrm{O}(9)$ & $2.553(11)$ \\
\hline $\mathrm{U}(5)-\mathrm{O}(17)$ & $2.723(11)$ & $\mathrm{Ca}(1)-\mathrm{OW}(30)$ & $2.587(13)$ \\
\hline$<\mathrm{U}(5)-\mathrm{O}_{\mathrm{ur}}>$ & 1.79 & $\mathrm{Ca}(1)-\mathrm{OW}(23)$ & $2.590(11)$ \\
\hline \multirow[t]{2}{*}{$<\mathrm{U}(5)-\mathrm{O}_{\mathrm{eq}}>$} & 2.50 & $\mathrm{Ca}(1)-\mathrm{OW}(28)$ & $2.882(12)$ \\
\hline & & $<\mathrm{Ca}(1)-\varphi>$ & 2.54 \\
\hline
\end{tabular}

$\mathrm{a}=-\mathrm{x}+1, \mathrm{y}-1 / 2,-\mathrm{z}+3 / 2$

$\mathrm{b}=-\mathrm{x},-\mathrm{y}+1,-\mathrm{z}+1$

$\mathrm{c}=-\mathrm{x}+1, \mathrm{y}+1 / 2,-\mathrm{z}+3 / 2$

$\mathrm{d}=-\mathrm{x}+2,-\mathrm{y}+1,-\mathrm{z}+2$

$\mathrm{e}=\mathrm{x}-1, \mathrm{y}, \mathrm{z}$

$\mathrm{f}=-\mathrm{x}+1,-\mathrm{y}+1,-\mathrm{z}+2$

$\mathrm{g}=-\mathrm{x}+1,-\mathrm{y}+1,-\mathrm{z}+$

$\mathrm{h}=\mathrm{x},-\mathrm{y}+3 / 2, \mathrm{z}-1 / 2$

$\mathrm{i}=\mathrm{x}+1, \mathrm{y}, \mathrm{z}$

$\mathrm{j}=\mathrm{x},-\mathrm{y}+3 / 2, \mathrm{z}+1 / 2$

$\varphi:\left(\mathrm{O}, \mathrm{H}_{2} \mathrm{O}\right)$ 
Deliens (1981). The refined atomic contents of the $\mathrm{Ba}(1)$ site are $96.8(6) \% \mathrm{Ba}$ and 3.2(6)\% $\mathrm{Ca}$, whereas the $\mathrm{Ba}(2)$ site contains $87.9(5) \% \mathrm{Ba}$ and $12.1(5) \% \mathrm{Ca}$. These results yield the empirical formula $\mathrm{Ca}_{2}\left(\mathrm{Ba}_{3.69} \mathrm{Ca}_{0.31}\right)$ $\left[\left(\mathrm{UO}_{2}\right)_{3} \mathrm{O}_{2}\left(\mathrm{PO}_{4}\right)_{2}\right]_{3}\left(\mathrm{H}_{2} \mathrm{O}\right)_{16}$, which may be idealized as $\mathrm{Ca}_{2} \mathrm{Ba}_{4}\left[\left(\mathrm{UO}_{2}\right)_{3} \mathrm{O}_{2}\left(\mathrm{PO}_{4}\right)_{2}\right]_{3}\left(\mathrm{H}_{2} \mathrm{O}\right)_{16}$, in excellent agreement with that reported by Piret \& Deliens (1981).

\section{Description of the Structure}

The structure of bergenite is shown projected along [010] in Figure 2. As with other structures of the phosphuranylite group (see Burns 1999), bergenite contains uranyl phosphate sheets of the phosphuranylite sheet anion-topology, with $\mathrm{H}_{2} \mathrm{O}$ groups and cations $(\mathrm{Ca}$ and $\mathrm{Ba}$ in this case) in the interlayer.

There are five symmetrically independent $U$ atoms in bergenite, each of which is part of an approximately linear $\left(\mathrm{UO}_{2}\right)^{2+}$ cation. The uranyl ions of $\mathrm{U}(1), \mathrm{U}(2)$ and $\mathrm{U}(3)$ are coordinated by five additional $\mathrm{O}$ atoms ar- ranged at the equatorial positions of pentagonal bipyramids, with the uranyl ion $\mathrm{O}$ atoms at the apices of the bipyramids, whereas the uranyl ions of U(4) and $\mathrm{U}(5)$ are coordinated in a similar fashion with six additional $\mathrm{O}$ atoms arranged at the equatorial positions of hexagonal bipyramids (Table 4).

The uranyl pentagonal bipyramids share equatorial edges with each other and with the hexagonal bipyramids to form chains that have a ratio of pentagonal bipyramids to hexagonal bipyramids of 2:1. Each of the three symmetrically distinct phosphate tetrahedra shares an edge with a hexagonal bipyramid, and its opposite corner with a pentagonal bipyramid, thus serving to link the offset chains of uranyl polyhedra into sheets (Fig. 3).

The interlayer of bergenite contains two symmetrically independent barium positions, one calcium position and eight distinct $\mathrm{H}_{2} \mathrm{O}$ groups. $\mathrm{Ba}(1)$ is in eleven-fold coordination with a mean $\mathrm{Ba}(1)-\varphi(\varphi$ : $\mathrm{O}$, $\left.\mathrm{H}_{2} \mathrm{O}\right)$ distance of $3.01 \AA$, and $\mathrm{Ba}(2)$ is in ten-fold coordination with a mean $\mathrm{Ba}(2)-\varphi$ distance of $2.95 \AA$. $\mathrm{Ba}(1)$

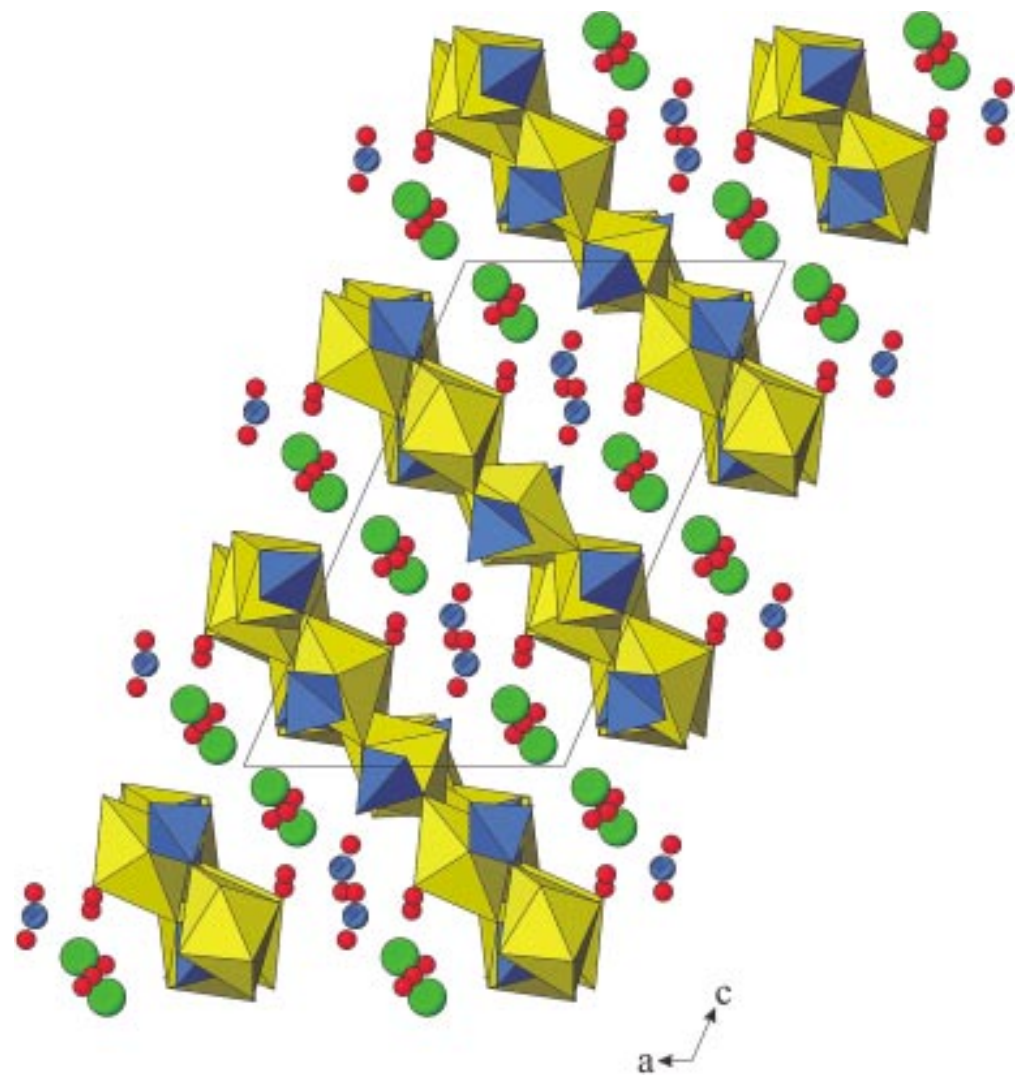

FIG. 2. Polyhedron representation of the crystal structure of bergenite projected along [010]. The uranyl polyhedra are shown in yellow, and the phosphate tetrahedra, in blue. The $\mathrm{Ba}$ atoms are shown as large green spheres, the $\mathrm{Ca}$ atoms as striped blue spheres, and the $\mathrm{H}_{2} \mathrm{O}$ groups as small red spheres. 
TABLE 5. BOND-VALENCE ANALYSIS FOR BERGENITE $(v u)^{*}$

\begin{tabular}{|c|c|c|c|c|c|c|c|c|c|c|c|c|}
\hline & $\mathrm{U}(1)$ & $\mathrm{U}(2)$ & $\mathrm{U}(3)$ & $\mathrm{U}(4)$ & $\mathrm{U}(5)$ & $\mathrm{P}(1)$ & $\mathrm{P}(2)$ & $\mathrm{P}(3)$ & $\mathrm{Ba}(1)$ & $\mathrm{Ba}(2)$ & $\mathrm{Ca}(1)$ & Total \\
\hline $\mathrm{O}(1)$ & 0.64 & 0.68 & & $0.73^{\times 2 \downarrow}$ & & & & & & & & 2.05 \\
\hline $\mathrm{O}(2)$ & & 1.72 & & & & & & & & 0.17 & & 1.88 \\
\hline$O(3)$ & 1.61 & & & & & & & & & 0.17 & & $\begin{array}{l}1.78 \\
1.55\end{array}$ \\
\hline$O(4)$ & & & & $1.55^{\times 2 \downarrow}$ & & & & & & & & $\begin{array}{l}1.55 \\
1.84\end{array}$ \\
\hline $\mathrm{O}(5)$ & 1.64 & & & & & & & & 0.20 & & & 1.84 \\
\hline O(6) & & & & & 1.65 & & & & $17^{\times 2 \downarrow}$ & & & $\begin{array}{l}1.65 \\
1.79\end{array}$ \\
\hline $\begin{array}{l}O(7) \\
O(8)\end{array}$ & & 0.53 & & & & 1.24 & & & $0.17^{-20}$ & & & $\begin{array}{l}1.99 \\
1.77\end{array}$ \\
\hline$O(9)$ & & 1.59 & & & & & & & 0.05 & & 0.21 & 1.85 \\
\hline$O(10)$ & 0.52 & & & & & & 1.30 & & & & & 1.82 \\
\hline$O(11)$ & & 0.52 & & & 0.36 & & 1.23 & & & & & 2.10 \\
\hline $\mathrm{O}(12)$ & & & $0.64^{\times 2 t}$ & & 0.68 & & & & 0.07 & & & 2.03 \\
\hline $\mathrm{O}(13)$ & 0.49 & & & $0.35^{\times 2 \downarrow}$ & & & & 1.19 & & & & 2.03 \\
\hline $\mathrm{O}(14)$ & 0.57 & 0.60 & & & 0.68 & & & & 0.13 & & & 1.98 \\
\hline$O(15)$ & & & 1.63 & & & & & & 0.06 & & 0.24 & 1.93 \\
\hline$O(16)$ & & & 0.55 & & & & & 1.33 & & & & 1.88 \\
\hline $\mathrm{O}(17)$ & 0.53 & & & & 0.26 & 1.24 & & & & & & 2.04 \\
\hline $\mathrm{O}(18)$ & & & 1.65 & & & & & & 0.22 & & & 1.87 \\
\hline OW(19) & & & & & & & & & 0.26 & & & 0.26 \\
\hline $\mathrm{O}(20)$ & & & 0.53 & & 0.36 & 1.19 & & & & & & 2.08 \\
\hline$O(21)$ & & & 0.47 & & 0.29 & & 1.23 & & & & & 2.00 \\
\hline $\mathrm{O}(22)$ & & & & & & 1.40 & & & & 0.36 & & 1.75 \\
\hline OW(23) & & & & & & & & & 0.22 & 0.04 & 0.19 & 0.45 \\
\hline $\mathrm{O}(24)$ & & & & & & & 1.33 & & & 0.28 & & 1.61 \\
\hline $\mathrm{O}(25)$ & & & & & & & & 1.32 & & 0.14 & 0.34 & 1.80 \\
\hline$O(26)$ & & 0.51 & & $0.24^{\times 2 \downarrow}$ & & & & 1.20 & & 0.08 & & 2.03 \\
\hline OW(27) & & & & & & & & & & & 0.27 & 0.27 \\
\hline OW(28) & & & & & & & & & 0.21 & & 0.08 & 0.29 \\
\hline OW(29) & & & & & & & & & & & 0.30 & 0.30 \\
\hline OW(30) & & & & & & & & & & 0.21 & 0.19 & 0.40 \\
\hline OW(31) & & & & & & & & & & 0.29 & & 0.29 \\
\hline OW(32) & & & & & & & & & & 0.16 & & 0.16 \\
\hline Total & 6.02 & 6.15 & 6.11 & 5.74 & 5.89 & 5.07 & 5.09 & 5.04 & 1.75 & 1.90 & 1.81 & \\
\hline
\end{tabular}

* Calculated with ideal occupancy of all atomic positions.

is coordinated mostly by the apical $\mathrm{O}$ atoms of uranyl ions (Table 4) and also by equatorial $\mathrm{O}$ atoms of uranyl polyhedra and interlayer $\mathrm{H}_{2} \mathrm{O}$ groups, whereas $\mathrm{Ba}(2)$ is coordinated by apical $\mathrm{O}$ atoms of phosphate tetrahedra, interlayer $\mathrm{H}_{2} \mathrm{O}$ groups and apical $\mathrm{O}$ atoms of uranyl ions. The eight-fold-coordinated $\mathrm{Ca}(1)$ is coordinated mostly by interlayer $\mathrm{H}_{2} \mathrm{O}$ groups and has a mean $\mathrm{Ca}(1)-\varphi$ distance of $2.54 \AA$ (Table 4 ).

\section{DiscUSSION}

The uranyl phosphate sheets in the minerals of the phosphuranylite group differ mainly in the orientations of their phosphate tetrahedra (Burns 1999), and may be divided on this basis (Fig. 4, Table 6) into four different geometrical isomers (Hawthorne 1983). In the vanmeersscheite-type sheet, found in vanmeersscheite and dumontite, the phosphate tetrahedra between the uranyl chains alternate orientations in an up-down up-down updown (ududud) pattern, and each pair of tetrahedra attached to a given uranyl hexagonal bipyramid has the same (S) orientation. This geometrical isomer may thus be referred to by the symbol udududS. In the phosphuranylite-type sheet, found in upalite, phosphuranylite,
françoisite-(Nd) and dewindtite, the tetrahedra change orientation in an up-up down-down up-up (uudduu) sequence, and the tetrahedra attached to the same uranyl hexagonal bipyramid have the same $(\mathrm{S})$ orientation, yielding the geometrical isomer uudduuS. The phurcalite-type sheet, found in phurcalite, phuralumite and althupite, has tetrahedra that also change orientations in an up-up down-down up-up (uudduu) pattern, but the tetrahedra attached to the same uranyl hexagonal bipyramid have opposite $(\mathrm{O})$ orientations, giving the geometrical isomer uudduuO. The uranyl phosphate sheet in bergenite is a novel geometrical isomer; the tetrahedra vary orientations in an up-up-down up-updown (uuduud) pattern, and the pairs of tetrahedra attached to uranyl hexagonal bipyramids change orientations in a complementary same-same-opposite (SSO) sequence, yielding the symbol uuduudSSO (Table 6).

\section{Comparison with previous work}

The unit cell presented in this work is one-third the volume of that reported by Piret \& Deliens (1981) on the basis of precession and Weissenberg photographs, 


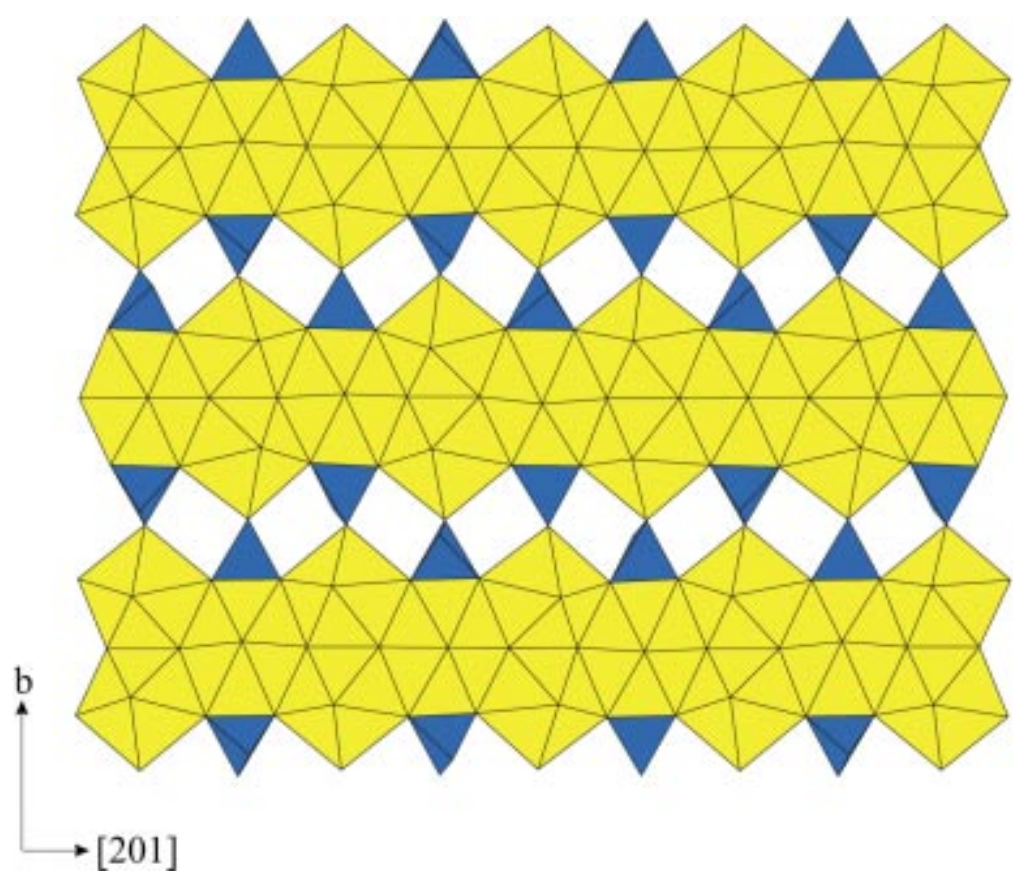

FIG. 3. The uranyl phosphate sheet of bergenite, projected normal to (102). The uranyl polyhedra are shown in yellow, and the phosphate tetrahedra, in blue.

TABLE 6. CELL PARAMETERS AND ORIENTATIONS OF PHOSPHURANYLITE-GROUP STRUCTURES

\begin{tabular}{|c|c|c|c|c|c|c|c|c|c|c|c|}
\hline Mineral & $\begin{array}{l}\text { Space } \\
\text { Group }\end{array}$ & $\begin{array}{c}a \\
(\AA)\end{array}$ & $\begin{array}{l}b \\
(\AA)\end{array}$ & $\begin{array}{c}c \\
(\AA)\end{array}$ & $\underset{\left({ }^{\circ}\right)}{\beta}$ & $\begin{array}{l}R 1 \\
(\%)\end{array}$ & OS & CD & $\begin{array}{l}\text { Geometrical } \\
\text { Isomer }\end{array}$ & Formula & Ref. \\
\hline $\begin{array}{l}\text { vanmeersscheite } \\
\text { dumontite }\end{array}$ & $\begin{array}{l}P 2_{1} m n \\
P 2_{1} / m\end{array}$ & $\begin{array}{r}17.06 \\
8.12\end{array}$ & $\begin{array}{l}16.76 \\
16.82\end{array}$ & $\begin{array}{l}7.02 \\
6.981\end{array}$ & 109.0 & $\begin{array}{r}12.6 \\
5.0\end{array}$ & $\begin{array}{l}(010) \\
(100)\end{array}$ & $\begin{array}{l}{[001]} \\
{[001]}\end{array}$ & $\begin{array}{l}\text { udududS } \\
\text { udududS }\end{array}$ & $\begin{array}{l}\mathrm{U}^{6+}\left[\left(\mathrm{UO}_{2}\right)_{3}(\mathrm{OH})_{2}\left(\mathrm{PO}_{4}\right)_{2}\right](\mathrm{OH})_{4}\left(\mathrm{H}_{2} \mathrm{O}\right)_{4} \\
\mathrm{~Pb}_{2}\left[\left(\mathrm{UO}_{2}\right)_{3} \mathrm{O}_{2}\left(\mathrm{PO}_{4}\right)_{2}\right]\left(\mathrm{H}_{2} \mathrm{O}\right)_{5}\end{array}$ & $\begin{array}{l}1 \\
2\end{array}$ \\
\hline $\begin{array}{l}\text { upalite } \\
\text { phosphuranylite } \\
\text { phosphuranylite } \\
\text { françoisite-(Nd) } \\
\text { dewindtite }\end{array}$ & $\begin{array}{l}P 2_{1} / a \\
C m c m \\
B m m b \\
P 2_{1} / c \\
B m m b\end{array}$ & $\begin{array}{r}13.70 \\
15.78 \\
15.83 \\
9.30 \\
16.03\end{array}$ & $\begin{array}{l}16.82 \\
13.70 \\
17.32 \\
15.61 \\
17.26\end{array}$ & $\begin{array}{l}9.331 \\
17.25 \\
13.72 \\
13.671 \\
13.61\end{array}$ & $\begin{array}{l}111.5 \\
112.8\end{array}$ & $\begin{array}{l}4.9 \\
3.6 \\
8.9 \\
6.1 \\
6.3\end{array}$ & $\begin{array}{l}(010) \\
(100) \\
(100) \\
(010) \\
(100)\end{array}$ & $\begin{array}{l}{[100]} \\
{[010]} \\
{[001]} \\
{[001]} \\
{[001]}\end{array}$ & $\begin{array}{l}\text { uudduuS } \\
\text { uudduuS } \\
\text { uudduuS } \\
\text { uudduuS } \\
\text { uudduuS }\end{array}$ & $\begin{array}{l}\mathrm{Al}\left[\left(\mathrm{UO}_{2}\right)_{3} \mathrm{O}(\mathrm{OH})\left(\mathrm{PO}_{4}\right)_{2}\right]\left(\mathrm{H}_{2} \mathrm{O}\right)_{7} \\
\left(\mathrm{H}_{3} \mathrm{O}\right)_{3} \mathrm{KCa}\left(\mathrm{UO}_{2}\right)\left[\left(\mathrm{UO}_{2}\right)_{3} \mathrm{O}_{2}\left(\mathrm{PO}_{4}\right)_{2}\right]_{2}\left(\mathrm{H}_{2} \mathrm{O}\right)_{8} \\
\mathrm{Ca}\left(\mathrm{UO}_{2}\right)\left[\left(\mathrm{UO}_{2}\right)_{3}(\mathrm{OH})_{2}\left(\mathrm{PO}_{4}\right)_{2}\right]_{2}\left(\mathrm{H}_{2} \mathrm{O}\right)_{12} \\
(\mathrm{Nd}, R E E)\left[\left(\mathrm{UO}_{2}\right)_{3} \mathrm{O}(\mathrm{OH})\left(\mathrm{PO}_{4}\right)_{2}\right]\left(\mathrm{H}_{2} \mathrm{O}\right)_{6} \\
\mathrm{~Pb}_{3}\left[\left(\mathrm{UO}_{2}\right)_{3}(\mathrm{OH}) \mathrm{O}\left(\mathrm{PO}_{4}\right)_{2}\right]_{2}\left(\mathrm{H}_{2} \mathrm{O}\right)_{12}\end{array}$ & $\begin{array}{l}3 \\
4 \\
5 \\
6 \\
7\end{array}$ \\
\hline $\begin{array}{l}\text { phurcalite } \\
\text { phuralumite } \\
\text { althupite* }\end{array}$ & $\begin{array}{l}P b c a \\
P 2_{1} / a \\
P 1\end{array}$ & $\begin{array}{l}17.42 \\
13.84 \\
10.95\end{array}$ & $\begin{array}{l}16.04 \\
20.92 \\
18.57\end{array}$ & $\begin{array}{c}13.60 \\
9.431 \\
13.50\end{array}$ & $\begin{array}{r}112.4 \\
68.2\end{array}$ & $\begin{array}{l}3.8 \\
47.0 \\
2 \quad 8.2\end{array}$ & $\begin{array}{l}(010) \\
(010) \\
(100)\end{array}$ & $\begin{array}{l}{[001]} \\
{[100]} \\
{[012]}\end{array}$ & $\begin{array}{l}\text { uudduuO } \\
\text { uudduuO } \\
\text { uudduuO }\end{array}$ & $\begin{array}{l}\mathrm{Ca}_{2}\left[\left(\mathrm{UO}_{2}\right)_{3} \mathrm{O}_{2}\left(\mathrm{PO}_{4}\right)_{2}\right]\left(\mathrm{H}_{2} \mathrm{O}\right)_{7} \\
\mathrm{Al}_{2}\left[\left(\mathrm{UO}_{2}\right)_{3}(\mathrm{OH})_{2}\left(\mathrm{PO}_{4}\right)_{2}\right](\mathrm{OH})_{4}\left(\mathrm{H}_{2} \mathrm{O}\right)_{10} \\
\mathrm{AlTh}\left(\mathrm{UO}_{2}\right)\left[\left(\mathrm{UO}_{2}\right)_{3} \mathrm{O}(\mathrm{OH})\left(\mathrm{PO}_{4}\right)_{2}\right]_{2}(\mathrm{OH})_{3}\left(\mathrm{H}_{2} \mathrm{O}\right)_{15}\end{array}$ & $\begin{array}{r}8 \\
9 \\
10\end{array}$ \\
\hline bergenite & $P 22_{1} / c$ & 10.09 & 17.24 & 17.351 & 113.7 & 75.0 & $(\overline{1} 02)$ & [201] & uuduudSSO & $\mathrm{Ca}_{2} \mathrm{Ba}_{4}\left[\left(\mathrm{UO}_{2}\right)_{3} \mathrm{O}_{2}\left(\mathrm{PO}_{4}\right)_{2}\right]_{3}\left(\mathrm{H}_{2} \mathrm{O}\right)_{16}$ & 11 \\
\hline
\end{tabular}

* althupite: $\alpha 72.6^{\circ}, \gamma 84.2^{\circ}$. References: (1) Piret \& Deliens (1982), (2) Piret \& Piret-Meunier (1988), (3) Piret \& Declercq (1983), (4) Demartin et al. (1991), (5) Piret \& Piret-Meunier (1991), (6) Piret et al. (1988), (7) Piret et al. (1990), (8) Atencio et al. (1991), (9) Piret et al. (1979), (10) Piret \& Deliens (1987), (11) this work. OS: orientation of sheet, CD: chain direction.

and can be transformed to the larger cell with the matrix [101/010/201]. The relationship between the two unit cells is shown in Figure 5, along with the corresponding changes in the optical orientation of bergenite.
The powder X-ray-diffraction pattern of bergenite was calculated using the program ATOMS version 5.1 (Dowty 2000) and the structural parameters were derived from the results of the single-crystal structure re- 


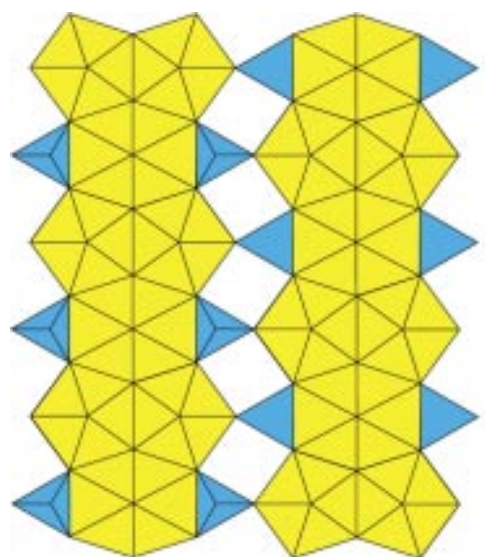

udududS

(a)

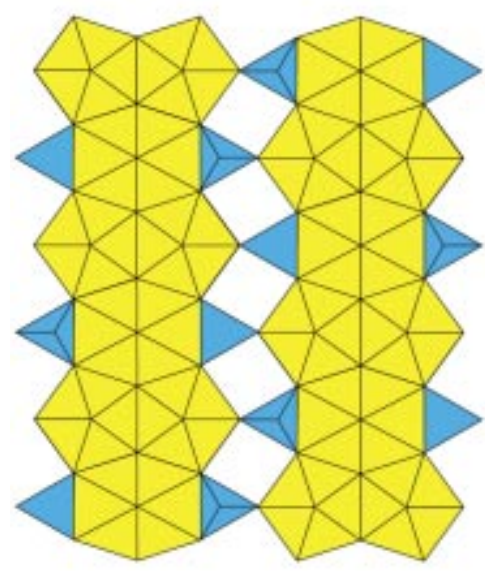

uudduuO

(c)

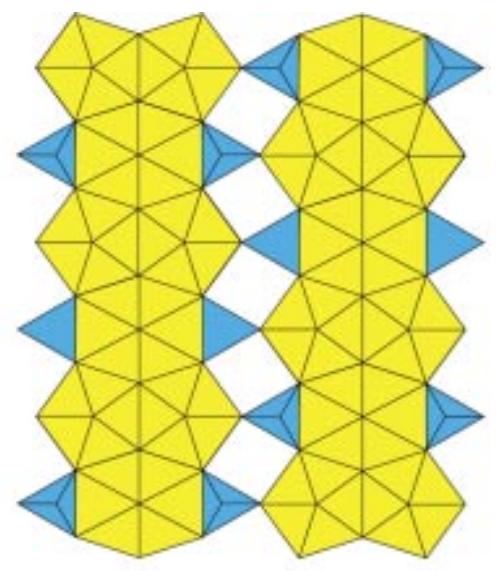

uudduuS

(b)

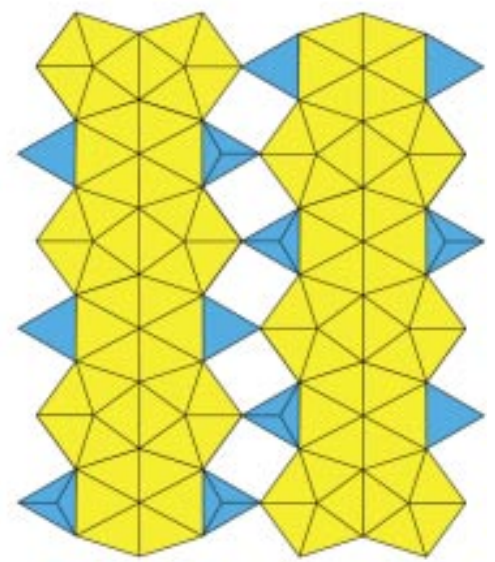

uuduudSSO

FIG. 4. Geometrical isomers of the uranyl phosphate sheet in members of the phosphuranylite group: (a) the vanmeersscheite-type sheet, in which tetrahedra between uranyl chains alternate orientations in an up-down up-down up-down (ududud) pattern, and each pair of tetrahedra attached to a given uranyl hexagonal bipyramid has the same (S) orientation; (b) the phosphuranylite-type sheet, in which tetrahedra change orientations in an up-up down-down up-up (uudduu) pattern, and the pair of tetrahedra attached to the same uranyl hexagonal bipyramid have the same (S) orientation; (c) the phurcalite-type sheet, in which tetrahedra change orientations in an up-up down-down up-up (uudduu) pattern, and the tetrahedra attached to the same uranyl hexagonal bipyramid have opposite $(\mathrm{O})$ orientations; $(\mathrm{d})$ the sheet that occurs in bergenite, in which tetrahedra change orientations in an up-up-down up-up-down (uuduud) pattern, and the pairs of tetrahedra attached to uranyl hexagonal bipyramids vary orientations in a complementary same-same-opposite (SSO) sequence. 
finement (Table 7). The simulated pattern yielded $d$ values whose positions are in agreement with the powder X-ray-diffraction data for bergenite of Piret \& Deliens (1981) [PDF 43-0668] and Bültemann \& Moh (1956) [PDF 20-0154], but differ in the low-angle reflections from the "barium-phosphuranylite" of Ross (1956).

\section{ACKNOWLEDGEMENTS}

We thank Michel Deliens and l'Institut Royal des Sciences Naturelles de Belgique for the loan of the specimen, and are grateful to him and to Luc André of the Musée Royal de l'Afrique Centrale for their hospitality during our recent visit to Belgium. Sergey

TABLE 7. COMPARISON OF POWDER X-RAY-DIFFRACTION DATA FOR BERGENITE AND "BARIUM-PHOSPHURANYLITE"

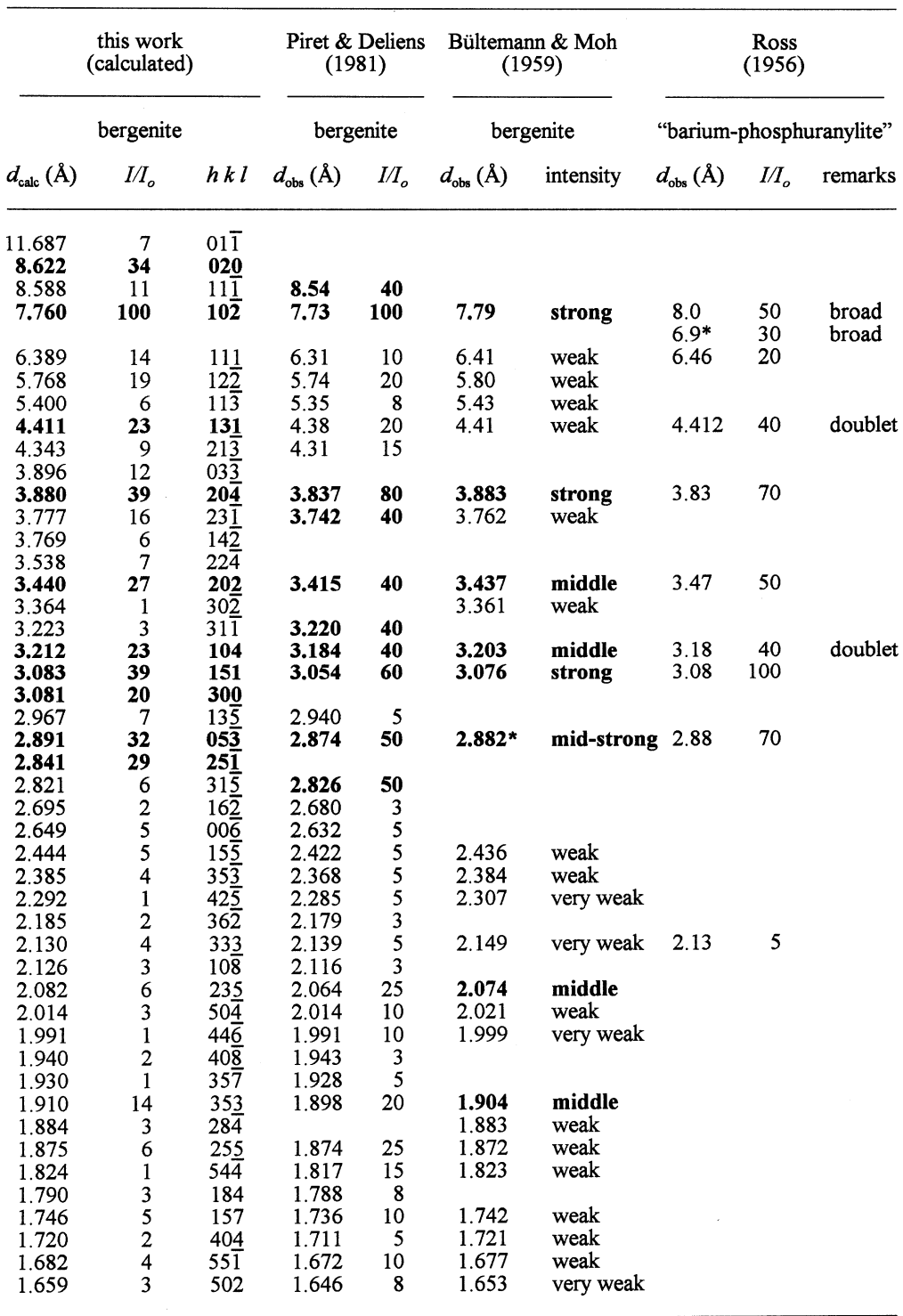

* corrected from the original reference. 


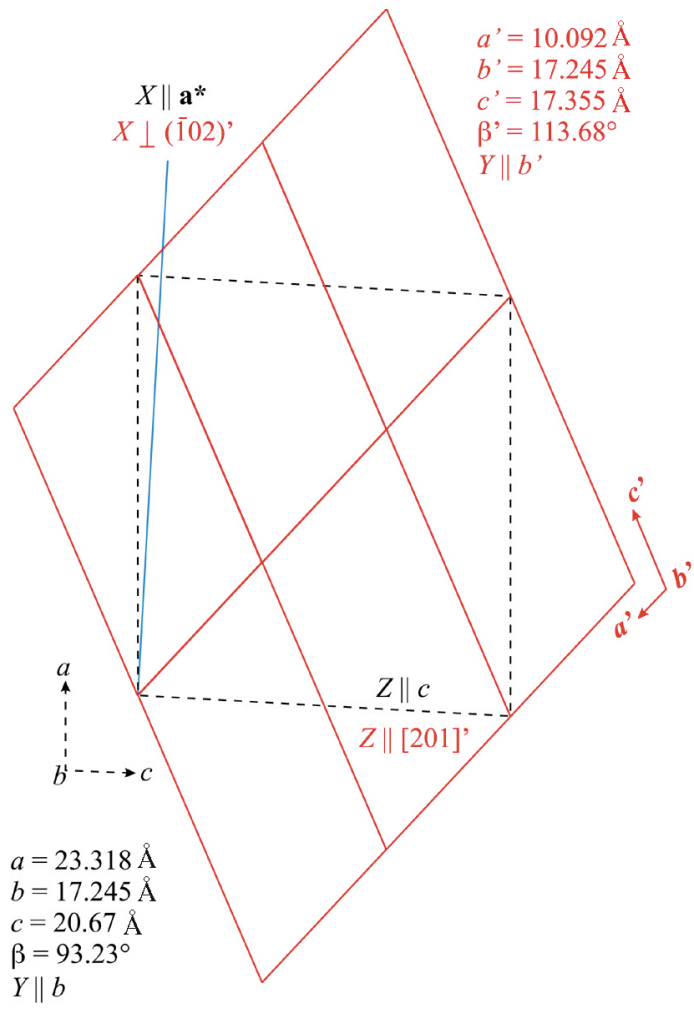

FIG. 5. Projection along [010] of the relationship between the large unit-cell of Piret $\&$ Deliens (1981), $a b c \beta$ (shown in black stipple), and the smaller unit cell, $a^{\prime} b^{\prime} c^{\prime} \beta^{\prime}$ (shown in red and denoted by ') presented in this work. The $X$ direction is shown in blue. The optical orientation for the previous large cell is: $Z$ parallel to $c, Y$ parallel to $b$, and $X$ parallel to $\mathbf{a}^{*}$. The optical orientation for the new small cell is: $Z$ parallel to [201]', $Y$ parallel to $b^{\prime}$, and $X$ perpendicular to (102)'.

Krivovichev is thanked for his assistance with the cell transformations and for his comments on the manuscript. We thank William Kinman and Jinesh Jain for assistance with the acquisition of the electron-microprobe data. The authors thank the reviewers: Francesco Demartin, Tullio Pilati, Carlo Maria Gramaccioli and Robert Finch, and the editor, Robert F. Martin, for their comments on the manuscript. AJL thanks the Mineralogical Association of Canada for the MAC Foundation 2001 Scholarship. This research was supported by the Environmental Management Science Program of the Office of Science, U.S. Department of Energy, grant DE-FGO7-97ER14820.

\section{REFERENCES}

Atencio, D., Neumann, R., Silva, A.J.G.C. \& Mascarenhas, Y.P. (1991): Phurcalite from Perus, São Paulo, Brazil, and redetermination of its crystal structure. Can. Mineral. 29, 95-105.

Brown, I.D. \& Altermatt, D. (1985): Bond-valence parameters obtained from a systematic analysis of the inorganic crystal structure database. Acta Crystallogr. B41, 244-247.

Buck, E.C., Brown, N.R., AND Dietz, N.L. (1996): Contaminant uranium phases and leaching at the Fernald Site in Ohio. Environ. Sci. Technol. 30, 81-88.

Bültemann, H.W. \& Moh, G.H. (1959): Bergenit, ein neues Mineral der Phosphuranylit Gruppe. Neues Jahrb. Mineral., Monatsh., 232-233.

BuRns, P.C. (1998): CCD X-ray area detectors applied to the analysis of mineral structures. Can. Mineral. 36, 847-853.

(1999): The crystal chemistry of uranium. In Uranium: Mineralogy, Geochemistry and the Environment (P.C. Burns \& R. Finch, eds.). Rev. Mineral. 38, 23-90.

Ewing, R.C. \& Hawthorne, F.C. (1997): The crystal chemistry of hexavalent uranium: polyhedron geometries, bond-valence parameters, and polymerization of polyhedra. Can. Mineral. 35, 1551-1570.

Demartin, F., Diella, V., Donzelli, S., Gramaccioli, C.M. \& Pilati, T. (1991): The importance of accurate crystal structure determination of uranium minerals. I. Phosphuranylite $\mathrm{KCa}\left(\mathrm{H}_{3} \mathrm{O}\right)_{3}\left(\mathrm{UO}_{2}\right)_{7}\left(\mathrm{PO}_{4}\right)_{4} \mathrm{O}_{4} \bullet 8 \mathrm{H}_{2} \mathrm{O}$. Acta Crystal$\log$. B47, 439-446.

DowTy, E. (2000): ATOMS for Windows and Macintosh, version 5.1, Shape Software, Kingsport, Tennessee.

Finch, R. \& Murakami, T. (1999): Systematics and paragenesis of uranium minerals. In Uranium: Mineralogy, Geochemistry and the Environment (P.C. Burns \& R. Finch, eds.). Rev. Mineral. 38, 91-180.

HAwTHORne, F.C. (1983): Graphical enumeration of polyhedral clusters. Acta Crystallogr. A39, 724-736.

Ungaretti, L. \& Oberti, R. (1995): Site populations in minerals: terminology and presentation of results of crystal structure refinement. Can. Mineral. 33, 907-911.

Ibers, J.A. \& Hamilton, W.C., eds. (1974): International Tables for X-ray Crystallography IV. The Kynoch Press, Birmingham, U.K.

LeE, D.J., SAng, K.N. \& LeE, S.R. (1982): Mineralogy of graphitic uranium ore in the Black Slate Formation of the Ogcheon Group [Korea]. Chosa Yongu Pogo - Chawon Kaepal Yonguso 13, 167-181 (in Korean). 
LOCOCK, A.J. \& BuRns, P.C. (2002a): The crystal structure of triuranyl diphosphate tetrahydrate. J. Solid State Chem. 163, 275-280.

$\&$ (2002b): Crystal structures of three framework alkali metal uranyl phosphate hydrates. J. Solid State Chem. 167, 226-236.

$\&$

(2003): The crystal structure of synthetic autunite, $\mathrm{Ca}\left[\left(\mathrm{UO}_{2}\right)\left(\mathrm{PO}_{4}\right)\right]_{2}\left(\mathrm{H}_{2} \mathrm{O}\right)_{11}$. Am. Mineral. 88, 240-244.

Murakami, T., Ohnuki, T., Isobe, H. \& Tsutomu, T. (1997): Mobility of uranium during weathering. Am. Mineral. 82, 888-899.

Piret, P. \& DeClercQ, J.-P. (1983): Structure cristalline de l'upalite $\mathrm{Al}\left[\left(\mathrm{UO}_{2}\right)_{3} \mathrm{O}(\mathrm{OH})\left(\mathrm{PO}_{4}\right)_{2}\right] \cdot 7 \mathrm{H}_{2} \mathrm{O}$. Un exemple de macle mimétique. Bull. Minéral. 106, 383-389.

\& Deliens, M. (1981): Nouvelles données sur la bergenite holotype. Bull. Minéral. 104, 16-18.

\& (1982): La vanmeersscheite $\mathrm{U}\left(\mathrm{UO}_{2}\right)_{3}$ $\left(\mathrm{PO}_{4}\right)_{2}(\mathrm{OH})_{6} \bullet 4 \mathrm{H}_{2} \mathrm{O}$ et la méta-vanmeersscheite $\mathrm{U}\left(\mathrm{UO}_{2}\right)_{3}$ $\left(\mathrm{PO}_{4}\right)_{2}(\mathrm{OH})_{6} \cdot 2 \mathrm{H}_{2} \mathrm{O}$, nouveaux minéraux. Bull. Minéral. 105, 125-128.

$\&$ (1987): Les phosphates d'uranyle et d'aluminium de Kobokobo. IX. L'althupite, $\operatorname{AlTh}\left(\mathrm{UO}_{2}\right)$ $\left[\left(\mathrm{UO}_{2}\right)_{3} \mathrm{O}(\mathrm{OH})\left(\mathrm{PO}_{4}\right)_{2}\right]_{2}(\mathrm{OH})_{3} \bullet 15 \mathrm{H}_{2} \mathrm{O}$, nouveau minéral; propriétés et structure cristalline. Bull. Minéral. 110, 6572.

\& Piret-Meunier, J. (1988): La françoisite-(Nd), nouveau phosphate d'uranyle et de terres rares; propriétés et structure cristalline. Bull. Minéral. 111, 443-449.
\& Piret-Meunier, J. (1988): Nouvelle détermination de la structure cristalline de la dumontite $\mathrm{Pb}_{2}\left[\left(\mathrm{UO}_{2}\right)_{3}\right.$ $\left.\mathrm{O}_{2}\left(\mathrm{PO}_{4}\right)_{2}\right] \cdot 5 \mathrm{H}_{2} \mathrm{O}$. Bull. Minéral. 111, 439-442.

$\&$

(1991): Composition chimique et structure cristalline de la phosphuranylite $\mathrm{Ca}\left(\mathrm{UO}_{2}\right)\left[\left(\mathrm{UO}_{2}\right)_{3}\right.$ $\left.(\mathrm{OH})_{2}\left(\mathrm{PO}_{4}\right)_{2}\right]_{2} \bullet 12 \mathrm{H}_{2} \mathrm{O}$. Eur. J. Mineral. 3, 69-77.

\& DECLERCQ, J.-P. (1979): Structure of phuralumite. Acta Crystallogr. B35, 1880-1882.

\& Deliens, M. (1990): Composition chimique et structure cristalline de la dewindtite $\mathrm{Pb}_{3}$ $\left[\mathrm{H}\left(\mathrm{UO}_{2}\right)_{3} \mathrm{O}_{2}\left(\mathrm{PO}_{4}\right)_{2}\right]_{2} \bullet 12 \mathrm{H}_{2} \mathrm{O}$. Eur. J. Mineral. 2, 399-405.

RoH, Y., Lee, S.R., Choi, S.K., Elless, M.P. \& Lee, S.Y. (2000): Physicochemical and mineralogical characterization of uranium contaminated soils. Soil and Sediment Contamination 9, 463-486.

Ross, V. (1956) Studies of uranium minerals. XXII. Synthetic calcium and lead uranyl phosphate minerals. Am. Mineral. 41, 915-926.

SOWder, A.G., Clark, S.B. \& FJELD, R.A. (1996): The effect of silica and phosphate on the transformation of schoepite to becquerelite and other uranyl phases. Radiochim. Acta 74, 45-49.

WALENTA, K. (1985): Uranotungstite, a new secondary uranium mineral from the Black Forest. Tschermaks Mineral. Petrogr. Mitt. 34, 25-34.

Received September 21, 2002, revised manuscript accepted January 29, 2003. 
